\title{
Plaque sealing by coronary angioplasty
}

\section{B Meier}

$\mathrm{T}$ he concept of plaque sealing by coronary balloon angioplasty has been around for some time. ${ }^{1}$ It is based on two principles. First, a coronary stenosis subjected to balloon angioplasty will not progress to a total occlusion later on unless it occludes abruptly during or immediately after the intervention. Even if a restenosis occurs, the smooth inner lining of the intimal proliferation virtually precludes endothelial rupture and thrombosis. Second, preventing plaque rupture and occlusive thrombosis impacts on the occurrence of myocardial infarction and death. Normalising the blood flow in a significantly stenosed coronary segment only addresses symptoms such as angina pectoris and perhaps exertional dyspnoea. The uncontested benefit of balloon angioplasty, namely alleviation of angina ${ }^{2}$ deals with a lifestyle problem (physical performance, elimination of medications) but not with the prognosis. Hence, restricting indications for coronary angioplasty to flow limiting lesions means barking up the wrong tree by ignoring what really counts-prevention of coronary occlusion and its devastating effects.

The plaque sealing principle has not been widely followed in spite of its theoretical appeal, mainly because of lack of documentation that balloon angioplasty indeed prevents subsequent myocardial infarction. For obvious reasons, such a strategy needs to be restricted to conspicuous and discrete narrowings in proximal segments of major coronary arteries. This may be a $60 \% 10 \mathrm{~mm}$ long stenosis in a proximal left anterior descending, dominant left circumflex, or large right coronary artery. Long lesions or lesions involving complex bifurcations may have a higher intervention risk than potential benefit over several subsequent years. Moreover, the principle cannot necessarily be extrapolated to coronary stenting with its small but significant bane of subacute thrombosis unknown to plain balloon angioplasty.

As outlined in the two previous reviews, the unstable plaque, the target of plaque sealing, exhibits a number of specific characteristics. The fibrous cap separating the plaque from the lumen is thin and eroded, at least in localised areas. It is lipid-rich with about $90 \%$ of macrophages being activated and half of them apoptotic. The intima contains only about $10 \%$ smooth muscle cells but many inflammatory cells. ${ }^{3}$ The collagen layers under the surface of the plaque are actively being destroyed by abundant matrix metalloproteinase, which again is stimulated by macrophages. ${ }^{4}$ Foam cells ${ }^{5}$ and activated angiotensin converting enzymes ${ }^{6}$ further destabilise the thin remaining protective layer.

It is exactly at these vulnerable zones where balloon angioplasty impacts first and most. It induces an immediate rupture of the thin fibrous cap. In contrast to spontaneous rupture this occurs under heparinisation and at a moment where coronary flow is optimised and observed. Therefore the risk of immediate thrombosis is only about $5 \%$ or $2 \%$ now that stents are available to remedy impending occlusions. Assuming that the subsequent risk for later infarction originating at this preventively dilated site is minute if not absent, this appears to be a favourable prognosis compared with the annual risk of infarction of a mild coronary lesion assessed at about $2 \%$ per year by the CASS (coronary artery surgery study) registry. ${ }^{7-9}$ Admittedly, this risk was assessed without the benefit of some of the pharmaceutical plaque pacifiers already discussed in the preceding articles, such as statins, thienopyridines, or angiotensin converting enzyme inhibitors.

The mechanism of plaque sealing and restenosis is the same. Intimal proliferation provides a new, smooth, and elastic coat to the ruptured plaque. This new cap may significantly reduce the lumen, hence cause restenosis, but it is not subject to the risk of rupture and thrombosis as was the brittle cap before balloon angioplasty. Smooth muscle cells are a prominent part of the new intima. They produce plaque smoothening proteins. ${ }^{10}$ They also strengthen the fibrous cap and favourably regulate the synthesis of interstitional collagen. ${ }^{11}$

\section{CLINICAL EVIDENCE OF EFFICACY OF MECHANICAL PLAQUE SEALING}

A Japanese study looked at 300 coronary sites angiographically at an average of seven years after balloon angioplasty. All these sites had been subjected to balloon angioplasty and found normally patent at a follow up angiogram six months after the intervention. Only two sites $(0.7 \%)$ were suspected to have caused a myocardial infarction during the seven year observation period. ${ }^{12}$ All other sites (99.3\%) were clearly identified as not being involved in the numerous infarctions that had occurred in the 249 patients since their initial angioplasty.

A retrospective analysis of over 3000 patients after successful balloon angioplasty proved that even the occurrence of restenosis did not increase mortality in the first six years of follow up..$^{13}$ This strongly suggests that even overshooting intimal proliferation lacks the risk of infarction as infarctions always translate to increased mortality.

In a large retrospective analysis on about 4000 patients undergoing balloon angioplasty complemented by stenting in $61 \%$ for lesions of various degrees of severity, the one year event rates of death, myocardial infarction, or repeat revascularisation did not differ between lesions of less than $50 \%$ diameter stenosis, lesions of $50-99 \%$ diameter stenosis, or total occlusions. Mortality and infarction rates were too low to be analysed separately. Thus, a benefit of plaque sealing of non-significant lesions could not be derived, but there was no disadvantage either. The one year follow up may have been too short to bring out the potential of plaque sealing. ${ }^{14}$

The DEFER trial was designed to prove that coronary angioplasty or stenting of a haemodynamically significant lesion is deleterious. ${ }^{15}$ About 200 patients with an angiographically identified lesion that had a normal fractional flow reserve $(>0.75)$ were randomised to undergo angioplasty (plaque sealing) with stent implantation in $46 \%$ or continued medical treatment. Event-free survival at 24 months did not differ between the two groups. Again the concept of plaque sealing was neither proved nor disproved, as the actively treated patients did not show an advantage at two years. 
Nonetheless, their lesions were taken care of whereas in the other patients the lesions were still there as a kind of Damoclean swords with their small but relevant potential for infarction or death. Incidentally, at least one of the two study related cardiac deaths in the conservative group occurred from a spontaneous plaque rupture of a medically treated non-significant lesion (personal communication). In addition the stenting in half the patients may have wiped out some of the potential benefits of mechanical plaque sealing due to the well recognised risk of subacute stent thrombosis of about $2 \%$. The infarction rate over two years in the group with angioplasty was $3 \%$ and the mortality rate $1 \%$, compared with $0 \%$ and $4 \%$, respectively, in the conservative group.

\section{SELECTION OF LESIONS FOR PLAQUE SEALING}

The individual risk of a stenosis to rupture, occlude, and produce a myocardial infarction is related to its severity. ${ }^{7}$ However, mild stenoses also harbour a certain risk for such events, albeit smaller than that of a severe stenosis. As there are usually many mild lesions to every severe lesion, it comes as no surprise that most infarctions are caused by mild lesions. A review of pertinent articles has shown that $68 \%$ of infarctions are caused by lesions that were recently assessed to be less than $50 \%$ and an additional $18 \%$ by $50-70 \%$ lesions. The remaining $14 \%$ are caused by the significant lesions that represent universally accepted targets for angioplasty. Hence, there is a potential to help prevent $84 \%$ of infarctions with mechanical means in addition to drugs and behavioural strategies already discussed. Any coronary lesion can produce a myocardial infarction at any time was the message of a seminal article published 15 years $\mathrm{ago}^{16}$ and again more recently. ${ }^{17}$ It makes sense to use balloon dilatations in proximally situated short lesions that at least raise the suspicion of haemodynamic significance (for example, at least $50 \%$ of diameter obstruction). The procedure should be done ad hoc during the diagnostic study to remain as cost efficient as possible. To terminate the diagnostic study and send the patient for an exercise test or a nuclear study does not make sense based on the concept that it is not the haemodynamic significance but the potential to cause infarction that provides the indication for angioplasty. If one or more significant lesions are treated at the same time, plaque sealing of one or more additional non-significant lesions becomes even more attractive as little additional material will be required that would not have been used anyway. Stents should be used when necessary but not invariably. It can be estimated that about 3\% of cases will show a menacing dissection that mandatorily needs to be stented. In an additional 30\%, the result may look suboptimal

Table 1 Methods to assess plaque vulnerability ${ }^{18-21}$

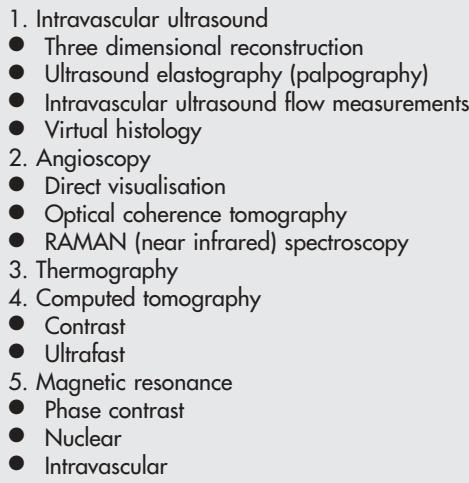

and stents will be inserted preventively. As we are dealing with mild and short lesions in large vessels, good and durable balloon alone results can be anticipated in about $90 \%$ without stenting. The comparative stent trials dealing with significant lesions cannot be translated to non-significant lesions without a conversion rate. Drug eluting stents do not really influence this recommendation, as they have at least as high a subacute thrombosis risk as bare stents, and because restenosis in mild lesions is already rare with balloon dilatation alone (about $10 \%$ ) or with a bare stent (about $5 \%$ ).

\section{PRELIMINARY ASSESSMENT OF VULNERABILITY OF THE PLAQUE}

Many methods have been advanced to assess the vulnerability of coronary plaques. The most discussed ones are listed in table $1 .^{18-21}$

None of these techniques have reached clinical application and few, if any, ever will. They are costly and flawed with some intrinsic complications. If a diagnostic angiogram showing a haemodynamically non-significant lesion meeting the requirements for plaque sealing enumerated above is complemented by one of the diagnostic studies in the table, there is scientific justification for it. However, there is little to gain. If the test proves the plaque to be unstable, the plaque sealing procedure will be performed anyway and the money spent on the diagnosis has been ill invested. If there is no evidence of vulnerability with one of the methods, perhaps another one will be used. If there is still no sign of vulnerability, angioplasty will be foregone for now. However, the passing of the lesion with diagnostic devices may have destabilised the formerly stable plaque or the plaque may turn unstable spontaneously at any time after the assessment (snapshot qualification). More money has already been spent than what would have been necessary to treat the lesion and the lesion is still not treated.

To follow the recommendation of people advocating invasive proof of haemodynamic significance for any interventional coronary procedure ${ }^{22}$ is even less attractive in terms of accomplishing the job with minimal investment of time and material. The plumbing part of percutaneous coronary interventions, consisting of re-establishing enough flow to prevent symptoms even at peak exercise, is important but should be only a secondary goal. The investment of an expensive flow or pressure wire to corroborate the significance of a stenosis can therefore not be recommended for routine cases. The money would be better invested in preventing subsequent infarction by balloon angioplasty and potent drugs.

\section{CONCLUSIONS}

In spite of lack of scientific proof, I should like to submit that plaque sealing by mechanical means is more important than plumbing in terms of prognosis of the patients, even though it can only be applied to a selection of stenoses and is far from being foolproof. A somewhat cynical statement by Spencer B King III, MD, on the occasion of a live angioplasty course in Atlanta, USA, on 29 May 2003 points in the same direction: "How to decide whether or not to dilate a mild lesion? If you want to dilate it, do IVUS (intravascular ultrasound); if you don't, do the flow wire!"

In a way, this is already happening time and time again, albeit not under the heading of plaque sealing. It is of some concern that angioplasty of mild lesions seems to be gaining ground only after the introduction of drug eluting stents. Stents, and that includes drug eluting stents, should play a minor role in mild lesions. Notwithstanding, it emerges that they will be used in all these cases and even cited as the justification to treat non-significant stenoses-that is, perform plaque sealing. 
Correspondence to: Professor Bernhard Meier, Swiss Cardiovascular Center Bern, University Hospital, 3010 Bern, Switzerland;

bernhard.meier@insel.ch

\section{REFERENCES}

1 Meier B, Ramamurthy S. Plaque sealing by coronary angioplasty. Cathet Cardiovasc Diagn 1995:36:295-7.

2 Rita-2 Trial Participants. Coronary angioplasty versus medical therapy for angina: the second randomised intervention treatment of angina (RITA-2) trial. Lancet 1997;350:461-8.

3 Libby P. Molecular bases of the acute coronary syndromes. Circulation 1995:91:2844-50.

4 Heistad DD. Unstable coronary-artery plaques. N Engl J Med 2003;349:2285-7.

5 Burke AP, Farb A, Malcom GT, et al. Coronary risk factors and plaque morphology in men with coronary disease who died suddenly. N Engl J Med 1997:336:1276-82.

6 Fukuhara M, Geary RL, Diz DI, et al. Angiotensin-converting enzyme expression in human carotid artery atherosclerosis. Hypertension 2000;35:353-9

7 Ellis S, Alderman E, Cain K, and the CASS investigators, et al. Prediction of risk of anterior myocardial infarction by lesion severity and measurement method of stenoses in the left anterior descending coronary distribution: a CASS registry study. J Am Coll Cardiol 1988;11:908-16.

8 Rozenman Y, Gilon D, Welber S, et al. Total coronary artery occlusion late after successful coronary angioplasty of moderately severe lesions: incidence and clinical manifestations. Cardiology 1994;85:222-8.

9 Hamon M, Bauters C, McFadden EP, et al. Six-month quantitative angiographic follow-up of $50 \%$ diameter stenoses dilated during multilesion percutaneous transluminal coronary angioplasty. Am J Cardio 1993:71:1226-9

10 Weissberg PL, Clesham GJ, Bennett MR. Is vascular smooth muscle cell proliferation beneficial? Lancet 1996;347:305-7.
11 Lafont A, Libby P. The smooth muscle cell: sinner or saint in restenosis and the acute coronary syndromes? J Am Coll Cardiol 1998;32:283-5.

12 Saito T, Date H, Taniguchi I, et al. Outcome of target sites escaping highgrade $(>70 \%)$ restenosis after percutaneous transluminal coronary angioplasty. Am J Cardiol 1999;83:857-61.

13 Weintraub WS, Ghazzal ZM, Douglas JS Jr, et al. Long-term clinical follow-up in patients with angiographic restudy after successful angioplasty. Circulation 1993;87:831-40.

14 Mercado N, Maier W, Boersma E, et al. Clinical and angiographic outcome of patients with mild coronary lesions treated with balloon angioplasty or coronary stenting. Implications for mechanical plaque sealing. Eur Heart $J$ 2003;24:541-51.

15 Bech GJW, De Bruyne B, Pijls NHJ, et al. Fractional flow reserve to determine the appropriateness of angioplasty in moderate coronary stenosis: a randomized trial. Circulation 2001;103:2928-34.

16 Little WC, Constantinescu M, Applegate RJ, et al. Can coronary angiography predict the site of a subsequent myocardial infarction in patients with mild-to-moderate coronary artery disease? Circulation 1988;78:1157-66.

17 Wilson RF. Assessing the severity of coronary-artery stenoses. N Engl J Med 1996;334:1735-7

18 Pasterkamp G, Falk E, Woutman $\mathrm{H}$, et al. Techniques characterizing the coronary atherosclerotic plaque: influence on clinical decision making? J Am Coll Cardiol 2000;36:13-21.

19 Badimon JJ, Fuster V. Can we image the "active" thrombus? Arterioscler Thromb Vasc Biol 2002;22:1753-4

20 Casscells W, Naghavi M, Willerson JT. Vulnerable atherosclerotic plaque: a multifocal disease. Circulation 2003;107:2072-5.

21 De Korte CL, Schaar JA, Mastik F, et al. Intravascular elastography: from bench to bedside. J Interv Cardiol 2003;16:253-9.

22 De Bruyne B, Bartunek J, Sys SU, et al. Simultaneous coronary pressure and flow velocity measurements in humans. Feasibility, reproducibility, and hemodynamic dependence of coronary flow velocity reserve, hyperemic flow versus pressure slope index, and fractional flow reserve. Circulation 1996;94:1842-9.

\section{IMAGES IN CARDIOLOGY}

\section{Cardiac papillary fibroelastoma of the mitral valve chordae}

P

apillary fibroelastoma of the mitral valve does not occur frequently, with fewer than 50 cases being reported in the literature. Therefore each case is interesting to report in order to improve the diagnosis and management of this uncommon tumour. We report an image of a fibroelastoma of the mitral valve chordae, without mitral insufficiency, discovered accidentally.

Papillary fibroelastoma was first described as gigantic Lambl's excrescences. In typical cases papillary fibroelastoma resembles a sea anemone with multiple papillary fronds attached to the endocardium by a short pedicle. Primary tumours of the heart and pericardium are extremely rare, with an incidence between $0.0017-0.28 \%$ in reported or collected necropsy series. More than $70 \%$ of these tumours are benign, and only $8 \%$ are papillary fibroelastomas, mostly located on heart valves. Cardiac primary fibroelastoma (CPFE) is by far the most common tumour arising from cardiac valves (about 70-80\%). CPFEs are small, avascular, benign tumours. The most frequent location is the valvar endocardium of the aortic valve $(30 \%)$ and the mitral valve (20-25\%) Only $10 \%$ are non-valvar. Because of their potential for cerebral and coronary embolisation, even small papillary fibroelastomas should be excised, even in asymptomatic patients.
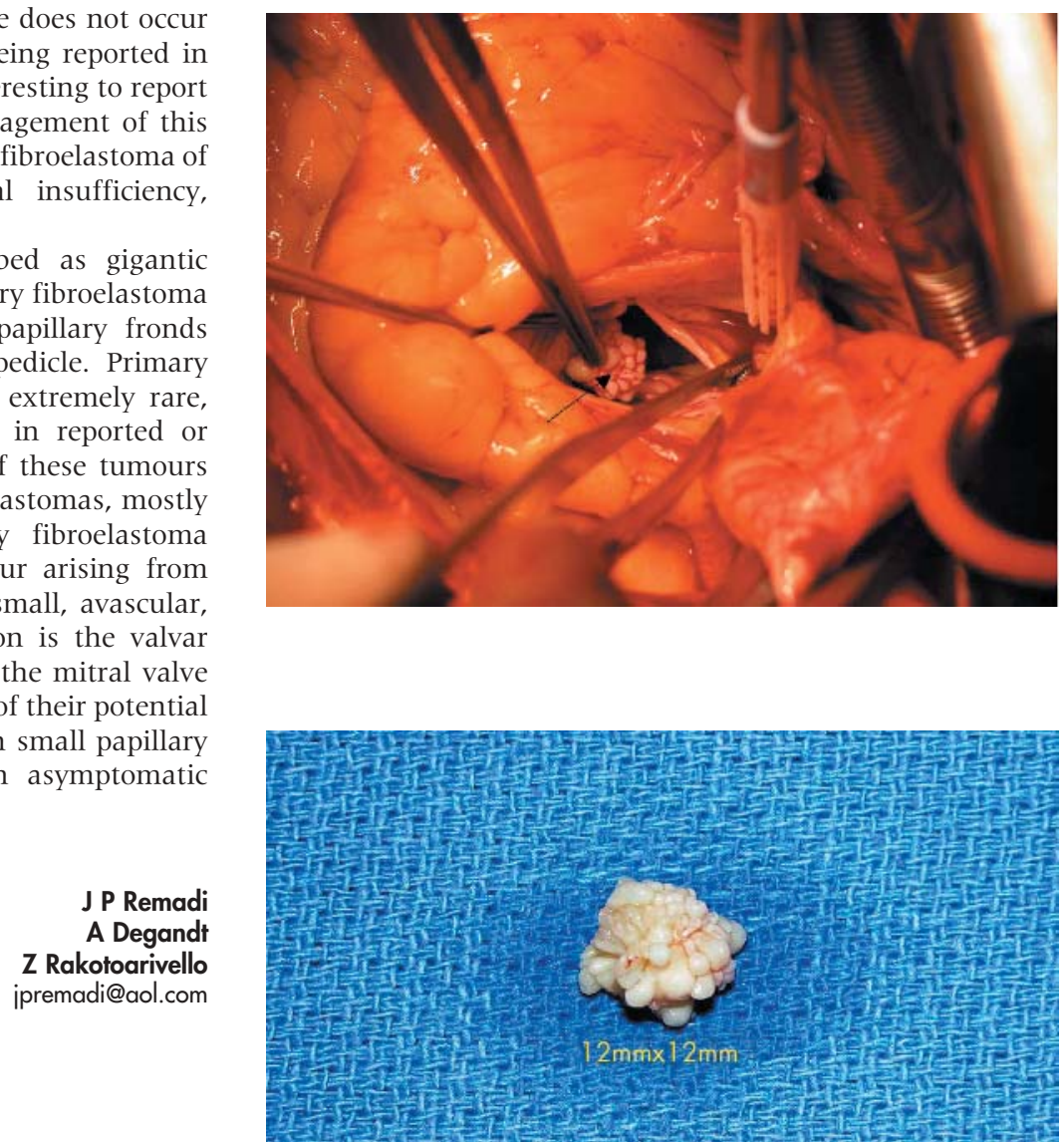